\title{
Algorithms for Detecting Clusters of Microcalcifications in Mammograms
}

\author{
Claudio Marrocco, Mario Molinara, and Francesco Tortorella \\ Dipartimento di Automazione, Elettromagnetismo, \\ Ingegneria dell'Informazione e Matematica Industriale \\ Università degli Studi di Cassino \\ 03043 Cassino (FR), Italy \\ \{c.marrocco, m.molinara, tortorella\}@unicas.it
}

\begin{abstract}
Mammography is a not invasive diagnostic technique widely used for early cancer detection in women breast. A particularly significant clue of such disease is the presence of clusters of microcalcifications. The automatic detection of such clusters is a very difficult task because of the small size of the microcalcifications and of the poor quality of the digital mammograms. In literature, all the proposed method for the automatic detection focus on the single microcalcification. In this paper, an approach that moves the final decision on the regions identified by the segmentation in the phase of clustering is proposed. To this aim, the output of a classifier on the single microcalcifications is used as input data in different clustering algorithms which produce the final decision. The approach has been successfully tested on a standard database of 40 mammographic images, publicly available.
\end{abstract}

\section{Introduction}

At present, mammography is the only radiological screening technique for detecting lesions in the breast using low doses of radiation. Moreover, it represents the only not invasive diagnostic technique that allows the diagnosis of a breast cancer at a very early stage, when it is still possible to successfully attack the disease with a suitable therapy. For this reason, for the female population at risk programs of wide mass screening via mammography have been carried out in many countries. One important clue of breast cancer is the presence of clustered microcalcifications. Microcalcifications are tiny granule-like deposits of calcium that appear on the mammogram as small bright spots. Their size ranges from about $0.1 \mathrm{~mm}$ to $0.7 \mathrm{~mm}$, while their shape is sometimes irregular. Isolated microcalcifications are not, in most cases, clinically significant. However, it is difficult for radiologists to provide both accurate and uniform evaluation for the enormous number of mammograms generated in widespread screening. In this case, a computer aided analysis could be very useful to the radiologist both for prompting suspect cases and for helping in the diagnostic decision as a "second reading". The goal is twofold [1]: to improve both the sensitivity of the diagnosis, i.e. the accuracy in recognizing all the actual clusters and its specificity, i.e. the ability to avoid erroneous detections. 
In the recent past, many approaches have been proposed for the automatic detection of clusters of microcalcifications [2-5], but all these methods focus on the analysis of the single microcalcifications. Generally speaking, all automatic systems are based on a two-step process: the first one is the segmentation of the mammogram while, in the second step, features derived from the segmentation are used as a basis for classifying each object as a microcalcification or as an artifact. Then, only in a following phase the filtered regions are clustered with very simple rules based on proximity of the microcalcifications, to individuate the clusters that are important for the diagnosis. However, the poor contrast on the mammogram makes microcalcifications not easily distinguishable from the mammal tissue in the background. These problems make the following feature extraction phase very critical and, moreover, errors on features will strongly affect the classification phase. As a consequence, the results of the classification can not be sufficiently reliable and the following clustering, even though very simple, can produce unsatisfactory results.

In our work we propose an alternative approach that, avoiding a decision on the single regions during the classification phase, considers the output of the classifier as a confidence degree of the single microcalcifications to be used in a successive phase of clustering. In this way, it is possible to create a suitable algorithm, that, reckoning with the spatial coordinates of the regions and the confidence degree, gives a possible partition in clusters of microcalcifications. In this way, the decision is taken on the whole cluster according to the characteristics of regions that it groups, thus leading to more reliable results. The confidence degree is so considered as an input feature for the clustering algorithm as it includes the knowledge obtained on all the extracted features in a set of non homogeneous data that represent (or are related to) the estimate of the a posteriori probability of the classifier.

In the rest of the paper we show, after a short description of the main clustering algorithms, how to adapt them to the particular situation of microcalcifications according to our method. A conclusive section describes the results obtained from experiments performed on real datasets and a comparison among the implemented algorithms.

\section{Basic Clustering Algorithms}

Clustering is a well known topic in the image processing field. Roughly speaking, clustering's goal is to achieve the best partition over a set of objects in terms of similarity. Similarity-base clustering is a simple technique that uses a similarity measure to guarantee if two objects are similar enough to put them in the same cluster. The similarity measure is usually defined through features of the object.

Clustering algorithms can be divided into two different categories according to the a priori knowledge on the problem that is analyzed: a first category (such as k-means) where it has to be specified the number of searched clusters and a second one where the number of clusters is variable according to the input data. Of course, the latter is more useful for our goal since we do not know anything about the presence of a cancer in the mammogram we are analyzing. Particularly, we focus on two different types of algorithms: hierarchical and sequential algorithms. 
First of all, it could be useful to give the definition of clustering [6]. Given a data set $\mathrm{X}=\left\{\mathrm{x}_{1}, \mathrm{x}_{2}, \ldots, \mathrm{x}_{\mathrm{N}}\right\}$ with $x_{i} \in R^{l}$, we define an $m$-clustering $\mathfrak{R}$ of $\mathrm{X}$, the partition of $\mathrm{X}$ in $m$ clusters, $C_{l}, \ldots, C_{m}$, so as the following conditions are satisfied:

- $C_{i} \neq 0, \quad \mathrm{i}=1, \ldots, \mathrm{m}$

- $\cup_{i=1}^{m} C_{i}=\mathbf{X}$

- $C_{i} \cap C_{j}=0, \quad \mathrm{i} \neq \mathrm{j}, \quad \mathrm{i}, \mathrm{j}=1, \ldots, \mathrm{m}$

In the algorithms for hierarchical clustering, a sequence of $\mathrm{N}$ nested clustering $\mathfrak{R}_{0} \subset \mathfrak{R}_{1} \subset \ldots \subset \mathfrak{R}_{\mathrm{N}-1}$ is produced where the index of the particular element in the sequence is said the level in the clustering sequence. In the agglomerative hierarchical clustering, any pair of samples grouped together at level $\mathrm{k}$ remain grouped together at all higher levels. In other words, two clusters $C_{p}$ and $C_{q}$ contained in clustering $\mathfrak{R}_{\mathrm{k}}$ are merged together in a single cluster present in $\mathfrak{\Re}_{\mathrm{k}+1}$; merging is realized according to a function $g\left(C_{i}, C_{j}\right)$ that is a similarity or dissimilarity measure between clusters. Each level is then characterized by means of an inconsistency coefficient [6] which measures the dissimilarity of clusters that are merged. Depending on the requirements of the application at hand, a threshold is set on the inconsistency coefficient so as to choose the level containing the most "natural" number of clusters.

A second approach is given by the sequential algorithms, in which the samples are sequentially considered and inserted in one of the clusters. Whereas hierarchical clustering algorithms must have all data present before clustering begins, in this type of algorithms the clustering can be performed even before the full data are present. The main drawback is that clustering depends strongly on the order of data presentation. One example of this approach is the leader clustering [7]: given a set of samples, we consider the first sample as the centre of a circular cluster of a specified radius. Then, if the second point falls in this region it belongs to the first cluster otherwise it is the centre of a new cluster. The same for the third point and so on until all the data have been considered. An useful variation to this approach is the leader-follower clustering [7] that consists of changing the cluster centre when new patterns enter in a cluster. In such algorithms, the properties of the produced clusters depend on the value chosen for the distance threshold (i.e. the radius of the circle): a large threshold leads to a small number of large clusters, while a small threshold to a large number of small clusters.

\section{Clustering Algorithms for Microcalcifications}

The purpose of this work is to study how to find clusters of microcalcifications in mammographic images. To this aim, the clustering algorithms described in section 2 have to be adapted to the particular problem we face. As previously said, features used in the clustering algorithms are the spatial coordinates $(\mathrm{x}, \mathrm{y})$ and the confidence degree $\mathrm{S}$.

\subsection{Hierarchical Approach}

In order to use a hierarchical algorithm $(H C)$, the idea is to consider the estimate $\mathrm{S}$ as a third coordinate in a three-dimensional space. Hence, the clustering is made on a 
feature vector of three elements to group not only points that are near (according to a similarity measure) in the $\mathrm{x}, \mathrm{y}$ plane but also on the third axis $\mathrm{z}=\mathrm{S}$.

In this way, for any pair of points $t_{i}=\left(x_{i}, y_{i}, z_{i}\right)$ and $t_{j}=\left(x_{j}, y_{j}, z_{j}\right)$ it is possible to define as similarity measure a weighted Euclidean distance:

$$
d\left(t_{i}, t_{j}\right)=\sqrt{\alpha\left[\left(x_{i}-x_{j}\right)^{2}+\left(y_{i}-y_{j}\right)^{2}\right]+\beta\left(z_{i}-z_{j}\right)^{2}}
$$

where $\alpha$ and $\beta$ are the weights used to normalize different data in a common range.

Then, using this similarity measure, it is possible to create a hierarchy of possible clustering and also to represent it with a dendrogram. The choice of the clustering is made using the inconsistency coefficient described in the previous section. When a clustering is chosen, it is possible to note that false clusters (i.e. clusters created by false regions) have a low medium value of the $\mathrm{z}$ component while this value is high for clusters created by true regions.

The choice of the weights is a critical point of this algorithm because we do not know how to relate the axes scales. Using the confidence degree we avoided a more problematic trouble, i.e. how to normalize all the different features; actually, we used only two weights, one for the spatial coordinates and another for the confidence degree but, referring to the sequential algorithms, it is possible to do not consider any weights.

\subsection{Sequential Approach}

The leader clustering $(L C)$, described in the previous section, needs to choose an order for the input data, because the clustering will strongly depend on it. To this aim, a possible approach is to order the points according to the confidence degree since regions that have a higher confidence degree can more probably represent microcalcifications. So, ordering data according to decreasing values of $S$, the first points that the algorithm will consider are those with a higher probability of being microcalcifications and, therefore, those with a higher probability of being grouped together.

Some limits of this algorithm are that it always considers a circular form for the cluster (and this is not true in the reality) and, moreover, that it is not sure that the region with a higher $\mathrm{S}$ is the centre of the cluster. To overcome these problems, in the previous section the leader-follower clustering has been described. Now, we propose a different approach, called Moving Leader $(M L C)$, that consists of considering as centre of the cluster a weighted centre of mass that has to be calculated each time a new region is grouped in the cluster. The used weight is the respective confidence degree $S_{i}$. Let us consider some points of coordinates $\mathbf{x}_{1}, \ldots, \mathbf{x}_{\mathrm{n}-1}$ with confidence degree respectively $\mathrm{s}_{1}, \ldots \mathrm{s}_{\mathrm{n}-1}$ grouped in a cluster; now, if we consider a sample $\mathbf{x}_{\mathbf{n}}$ with confidence degree $s_{n}$ that has to be grouped in that cluster, then, the new centre will be:

$$
\boldsymbol{w}_{\boldsymbol{n}}=\frac{\sum_{i=1}^{n} s_{i} \boldsymbol{x}_{\boldsymbol{i}}}{\sum_{i=1}^{n} s_{i}}=\frac{\sum_{i=1}^{n-1} s_{i} \boldsymbol{x}_{\boldsymbol{i}}+s_{n} \boldsymbol{x}_{\boldsymbol{n}}}{\sum_{i=1}^{n} s_{i}}=\frac{\sum_{i=1}^{n-1} s_{i}}{\sum_{i=1}^{n} s_{i}}\left(\frac{\sum_{i=1}^{n-1} s_{i} \boldsymbol{x}_{\boldsymbol{i}}}{\sum_{i=1}^{n-1} s_{i}}+\frac{s_{n}}{\sum_{i=1}^{n-1} s_{i}} \boldsymbol{x}_{\boldsymbol{n}}\right)
$$

In this way, the centre of the cluster moves towards the direction where the points are more dense and so, where there is a higher probability of finding new microcalcifications. Moreover, the shape of the new cluster is not a circle but it is created merg- 
ing $\mathrm{n}$ circles. For example in fig. 1.a, the point $\boldsymbol{w}_{1}$ is the centre of a cluster, when $\boldsymbol{x}_{2}$ has to be grouped in the cluster, the centre moves to $w_{2}$. The shape of the region that contains the final cluster is the union between the two circles (fig. 1.b). When a lot of points are analyzed we have a shape like in fig. 1.c.

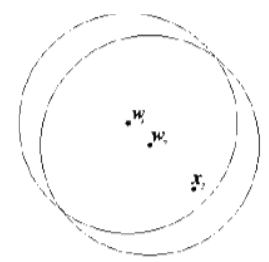

(a)

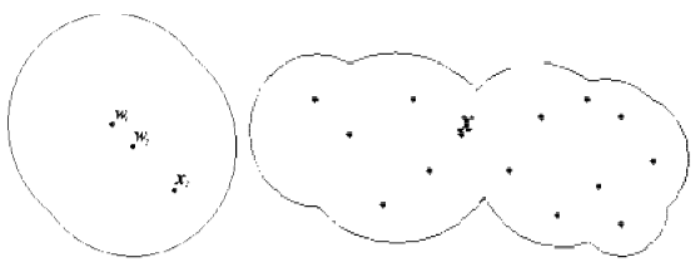

(b)

(c)

Fig. 1. (a) $w_{1}$ is the centre of a cluster, when $x_{2}$ has to be grouped in the cluster, the centre moves to $\boldsymbol{w}_{2}$ (b) the shape of the cluster is the union of two circles (c) an example of final shape of the cluster

When clusters have been created, a post-processing operation can be performed to better group microcalcifications. This strategy consists of merging two clusters that share at least one microcalcification. This approach, that we called Moving Leader with Merge (MLMC), let us join near regions avoiding an excessive number of clusters that is not corresponding to the reality.

\section{Experimental Results}

The system has been tested on a standard database provided by courtesy of the National Expert and Training Centre for Breast Cancer Screening and the Department of Radiology at the University of Nijmegen, the Netherlands. It contains 40 digitized mammographic images composed of both oblique and craniocaudal views from 21 patients. All images have a size of 2048x2048 pixels and use 12 bits per pixel for the gray levels. Each mammogram has one or more clusters of microcalcifications marked by radiologists; each cluster is described by the centre and the radius of a circle totally containing it. The total number of clusters is 105,76 of which are malignant and 29 benign.

As in [8], segmentation by Tree-Structured Markov Random Field (TS-MRF) and two-stage classification has been performed. Thus, for each region, we have at our disposal the coordinates and the output of an SVM classifier that indicates the signed distance of a region from the optimal separating hyperplane between the true and the false class. Using these input data, the clustering algorithms described in section 3 can be applied. For each algorithm, five different runs were executed according to an opportune parameter; for the hierarchical algorithm, the inconsistency coefficient has been varied from the $75 \%$ to the $95 \%$ of its maximum value with a $5 \%$ step, while for the sequential algorithms, a mean radius $\mathrm{R}_{\mathrm{m}}$ has been evaluated from all the clusters marked by radiologists and the experiments were performed using $R_{m}$ plus or minus the $10 \%$ and the $20 \%$ of its value. 
The performance of the proposed algorithms were only evaluated on the true positive clusters since we want to show how our method fits with the description of microcalcifications clusters. To highlight that, a dimensionless parameter, that we called covering factor $(C F)$, has been introduced; this takes into account if a cluster is not well recognized, i.e. if a cluster of the ground truth is recognized but it is subdivided in more than one sub-cluster or if an identified cluster is greater than the corresponding circle in the ground truth. For the $j$-th cluster in the ground truth, we have:

$$
C \boldsymbol{F}_{j}=\frac{\sum_{k=1}^{N_{j}} \boldsymbol{W}_{j k} \boldsymbol{I}_{j k}}{\boldsymbol{G}_{j}}
$$

where $N_{j}$ is the number of sub-clusters, $I_{j k}$ is the area of the intersection between the $k$-th sub-cluster and the $j$-th circle in the ground truth, $\mathrm{G}_{\mathrm{j}}$ is the area of that circle and $W_{j k}=I_{j k} /\left(N_{j}^{*} A_{j k}\right)$, with $A_{j k}$ area of the $k$-th sub-cluster, is a weight that reaches its maximum value, i.e. one, when there is only one sub-cluster totally contained in the circle of the ground truth. Fig. 2 shows how to evaluate the $\mathrm{CF}$ for a cluster subdivided into three sub-clusters, two totally contained in the circle of the ground truth and one that trespasses the boundary of the ground truth.

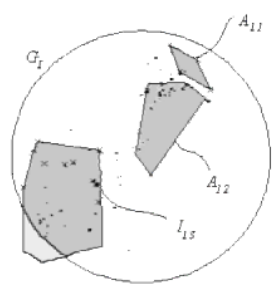

Fig. 2. Evaluation of the covering factor: in this case, $C F_{I}=\left(W_{11} I_{11}+W_{12} I_{12}+W_{13} I_{13}\right) / G_{1}$ where $I_{11}=A_{11}, I_{12}=A_{12}$ and $W_{11}=I_{11} / 3 A_{11}=1 / 3, W_{12}=I_{12} / 3 A_{12}=1 / 3, W_{13}=I_{13} / 3 A_{13}$.

However, we are comparing the cluster area evaluated by our method (that is based on the convex hull of regions) with the circles made by radiologists that are not very accurate. As a consequence, the CF never reaches high values, i.e. it is impossible that the estimated area is equal to the area of the circle in the ground truth. If this happens, it is due to a bad behaviour of the clustering algorithms that groups too big regions; this is especially visible when the inconsistency coefficient for the hierarchical clustering or the medium radius for the sequential algorithms becomes too great. An example is presented in fig. 3: fig. 3.a shows the best situation for a hierarchical algorithm with inconsistency coefficient equal to $85 \%$ while in fig. 3 .b we can see that a cluster is lost for an inconsistency coefficient equal to $95 \%$.

Thus, to better evaluate the performance of the algorithms, we also have to look at the number of clusters detected. Hence, we consider an algorithm better than another when, for the same number of detected clusters, it has the greater CF. The results obtained in terms of medium CF over all the images and percentage of detected clusters are reported in table 1.a for the hierarchical algorithm and in table 1.b for the sequential algorithms. 


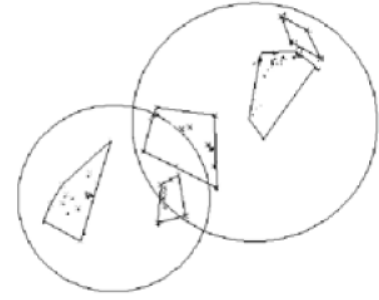

(a)

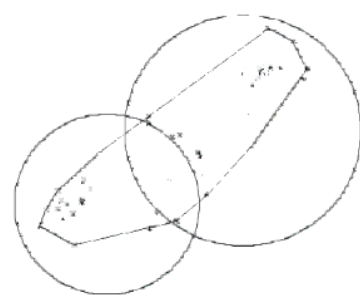

(b)

Fig. 3. Clustering of image c04c with a hierarchical algorithm: (a) with an inconsistency coefficient equal to $85 \%$ the two clusters are correctly recognized while (b) a cluster is lost with an inconsistency coefficient equal to $95 \%$

Table 1. Results obtained in terms of CF and percentage of detected clusters for the hierarchical (a) and the sequential (b) algorithms.

(a)

\begin{tabular}{|c|c|c|}
\cline { 2 - 3 } \multicolumn{1}{c|}{} & \multicolumn{2}{c|}{ HC } \\
\hline IC & CF & \% cl. \\
\hline $\mathbf{7 5 \%}$ & 0.09 & $94 \%$ \\
\hline $\mathbf{8 0 \%}$ & 0.11 & $96 \%$ \\
\hline $\mathbf{8 5 \%}$ & 0.12 & $96 \%$ \\
\hline $\mathbf{9 0 \%}$ & 0.15 & $94 \%$ \\
\hline $\mathbf{9 5 \%}$ & 0.19 & $94 \%$ \\
\hline
\end{tabular}

(b)

\begin{tabular}{|c|c|c|c|c|c|c|}
\cline { 2 - 7 } \multicolumn{1}{c|}{} & \multicolumn{2}{c|}{ LC } & \multicolumn{2}{c|}{ MLC } & \multicolumn{2}{c|}{ MLMC } \\
\hline $\mathbf{R}$ & $\mathbf{C F}$ & \%cl. & CF & \%cl. & CF & \%cl. \\
\hline $\mathbf{R}_{\mathrm{m}} \mathbf{- 2 0 \%}$ & 0.09 & $98 \%$ & 0.11 & $98 \%$ & 0.17 & $100 \%$ \\
\hline $\mathbf{R}_{\mathrm{m}} \mathbf{- 1 0 \%}$ & 0.12 & $100 \%$ & 0.13 & $100 \%$ & 0.20 & $100 \%$ \\
\hline $\mathbf{R}_{\mathrm{m}}$ & 0.14 & $100 \%$ & 0.15 & $100 \%$ & 0.21 & $100 \%$ \\
\hline $\mathbf{R}_{\mathrm{m}}+\mathbf{1 0 \%}$ & 0.15 & $100 \%$ & 0.16 & $98 \%$ & 0.24 & $98 \%$ \\
\hline $\mathbf{R}_{\mathrm{m}}+\mathbf{2 0 \%}$ & 0.18 & $98 \%$ & 0.19 & $96 \%$ & 0.28 & $98 \%$ \\
\hline
\end{tabular}

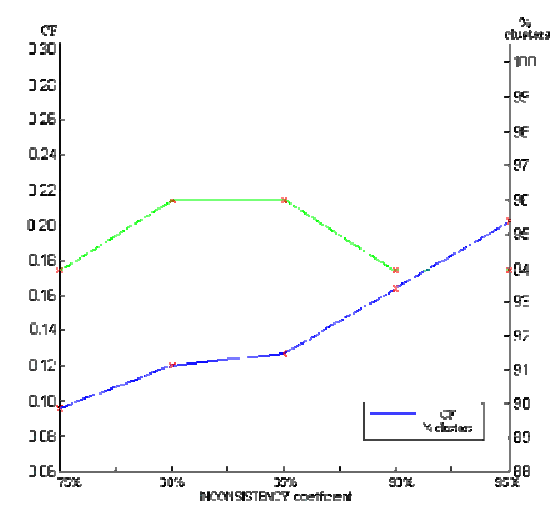

(a)

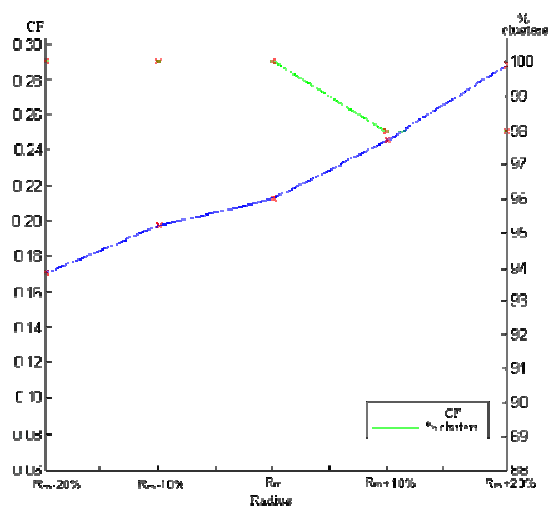

(b)

Fig. 4. Graphical results obtained in terms of $\mathrm{CF}$ and percentage of detected clusters as a function of the IC for the HC (a) and of the radius for the best sequential algorithm, i.e. the MLMC (b)

Then, in fig. 4 we report a graph of these results with two axes, one for the CF and one for the percentage of detected clusters. Fig. 4.a shows that the hierarchical algorithm never reaches a percentage of detection equal to $100 \%$, while in fig. $4 . \mathrm{b}$ 
we show the results for the MLMC that outperforms the other sequential algorithms. In fact, it reaches the $100 \%$ of detected clusters with a CF greater than all the other algorithms.

In summary, the experiments show that it is possible to improve the clustering of microcalcifications clusters, especially with the MLMC algorithm. It remains to test the algorithms on the clustering of the false regions, i.e. to demonstrate that the distribution of false positive does not influence the good capability of clustering shown by these algorithms.

\section{References}

1. De Santo, M., Molinara, M., Tortorella, F., Vento, M.: Automatic Classification of Clustered Microcalcifications by a Multiple Expert System. Pattern Recognition 36 (2003), 1467 -1477 .

2. Karssemeijer, N.: Adaptive Noise Equalization and Recognition of Microcalcification Clusters in Mammograms. International Journal of Pattern Recognition and Artificial Intelligence 7 (1993), 13571376.

3. Strickland, R.N., Hahn, H.: Wavelet Transforms for Detecting Microcalcifications in Mammograms. IEEE Transaction on Medical Imaging 15 (1996), 218-229.

4. Netsch, T., Peitgen, H.: Scale Space Signatures for the Detection of Clustered Microcalcifications in Digital Mammograms. IEEE Transaction on Medical Imaging 18, (1999), 774786.

5. Cheng, H.D., Lui, Y.M., Freimanis, R.I.: A Novel Approach to Microcalcification Detection Using Fuzzy Logic Technique. IEEE Transaction on Medical Imaging 17 (1998), 442-450.

6. Theodoridis, S., Koutroumbas, K.: Pattern Recognition, $2^{\text {nd }}$ Edition. Elsevier Science (2003).

7. Duda, R.O., Hart, P.E., Stork, D.G.: Pattern Classification, $2^{\text {nd }}$ Edition. John Wiley \& Sons, Inc. (2001).

8. D’Elia, C., Marrocco, C., Molinara, M., Poggi, G., Scarpa, G., Tortorella, F.: Detection of Microcalcifications Clusters in Mammograms through TS-MRF Segmentation and SVM-based Classification. Proceedings of the 17th International Conference on Pattern Recognition, Cambridge, UK, IEEE Computer Society Press (2004). 